\title{
TERRITORIO Y SOCIEDAD ENTRE LOS CAYAPAS DEL SIGLO XVI
}

Como ya anunciamos en ocasión anterior, ${ }^{1}$ en el presente trabajo nos proponemos analizar en profundidad las listas de bautismos incluidos en la Relación de fray Gaspar de Torres de 1598 que se refieren a poblaciones de cayapas, yambas, lachas, y malabas, habitantes de las cuencas de los ríos Mira, Mataje, Santiago, Cayapas y Onzole. Al parecer, los 1.484 individuos bautizados en los años 1595 y 1597 en esa región podrían pertenecer a tres o cuatro grupos étnicos y se hallarían agrupados formando linajes emparentados entre sí, cuyos caciques o principales encabezarían cada uno de los grupos a los que el propio Gaspar de Torres denomina «ayllus», haciendo así énfasis en el hecho de que tales grupos eran parientes en cierto modo.

Aunque el análisis de los nombres de las personas bautizadas no nos permita establecer el sistema de parentesco en términos muy precisos, esperamos poder fijar, con un cierto grado de seguridad, los agrupamientos étnicos y lingüísticos y su grado de mestizaje mutuo.

\section{LA ENTRADA DF: FRAY GASPAR DE TORRES}

Los trabajos de descubrimiento, pacificación y evangelización de los grupos indígenas de la región, pero especialmente de cayapas y lachas, se iniciaron con la llegada del doctor Juan del Barrio Sepúlveda, como oidor de la real audiencia de Quito, en octubre de 1596. Su experiencia como oidor, gobernador y capitán general

1 Palop, Josefina: Los cayapas en el siglos XVI. «Arqueología y Etnohistoria del Sur de Colombia y Norte del Ecuador», págs. 231-252. Guayaquil, 1986, pág. 246. 
en Santo Domingo (1576) y Panamá (1580-1596), le sirvió para enfrentar con prontitud y acierto muchos de los problemas de la audiencia de Quito. ${ }^{2}$ Por aquellas fechas, como lo había sido desck. 1535 y lo seguiría siendo por siglos, el problema de la comunicación entre Quito y la costa de Esmeraldas, era un problema de gran importancia desde el punto de vista económico y político, ${ }^{3}$ pero su resolución implicaba la «pacificación» o evangelización de los grupos indígenas que habitaban aquella región. De ahí el papel fundamental que desempeñaron fray Gaspar de Torres y Luis Gualapiango, el cacique de Lita, dirigidos hábilmente por el doctor Juan del Barrio Sepúlveda, en la apertura de un camino hacia la costa, a través del país habitado por los yumbos, lachas y cayapas, ruta que se conocería posteriormente con el nombre de camino de Malbucho, por un lugar de su itinerario.

Sobre fray Gaspar de Torres tenemos dos valiosas opiniones. De una parte fray Gabriel Téllez (Tirso de Molina), en su Crónica General de la Merced, al tratar del misionero dice: «Por una información que el P. Maestro Fray Gaspar de Prieto presentó al Real Consejo de Indias, es también manifiesto lo mucho que trabajó en la conversión de aquellos miserables»; ${ }^{4}$ por otra parte, el propio Juan del Barrio Sepúlveda, en carta al rey de 3 de marzo de 1598 decía de fray Gaspar que «es un buen frayle y por tal avido en su orden y de todos quantos le conocen, será a mi parecer de sesenta años, ágil y de buena condición y trato afable y aunque no es letrado sabe lo que baste a un buen sacerdote yn agilibus tiene experiencia y buena maña. Tengo en él, por las dichas raçones ordinaria correspondencia y le animo y regalo quanto puedo, con cartas a lo menos». ${ }^{5}$ Con tales condiciones personales la «pacificación» de los indios podía contarse como un hecho. Hacía falta, sin embargo, un mediador indígena. Este iba a ser Luis Gualapian-

2 Ibídem, pág. 232.

3 Alcina, José; Moreno, Encarnación y Peña, Remedios de la: Penetración espafiola en Esmeraldas (Ecuador): tipologia del descubrimiento. «Revista de Indias》. núms. 143-144, págs. 65-121. Madrid, 1976.

4 Monroy, Pray Joel: El Convento de la Merced de Quito, de 1534 a 1617 2." ed. Quito, 1938, pág. 307. fol. $3 r$.

5 A.G.I., Quito, 29. Carta de Juan del Barrio al Key, 3 de marzo de 1598. 
go, cacique del pueblo de Lita, posiblemente de origen carangue, que había dominado en primer lugar a los lachas ${ }^{6}$ y que pretendía seguir expandiendo su dominio al territorio de los cayapas. Según se desprende del «Asiento que el doctor Juan del Barrio Sepúlveda (...) tomó con estos caçiques para la nueva conversión a nuestra santa fee cathólica de estos infieles», tanto Luis Gualapiango, como su hijo Alonso, quienes ya tienen como amigos a los indios cayapas se comprometen a ayudar para que «se reduzcan a una o más poblaciones y reciban el agua del Santo baptismo y conozcan al Rey nuestro señor y sean vasallos de su Magestad, a cambio de que S. M. el rey dé a Alonso Gualapiango el nombramiento de Gobernador» de los indios que se poblaran en la provincia de Lita. ${ }^{7}$

La amistad de Luis Gualapiango y los cayapas provenía del hecho de que habiendo sido atacados en su antiguo asentamiento de Çampi por los mulatos de Esmeraldas, fueron auxiliados por Gualapiango quien con trescientos indios de Lita les defendió, ayudándolos a reinstalarse más al interior en el lugar de Pisuunto. Todo ello había sucedido en 1587 y desde entonces las relaciones entre Gualapiango y los cayapas habían sido excelentes.

La operación ideada y montada por el Dr. Juan del Barrio consistía en aprovechar estas circunstancias y, utilizando las habilidades de fray Gaspar de Torres, conseguir fundar dos poblaciones en tierras de cayapas -Espíritu Santo y Nuestra Señora de Guadalupe - que sirviesen de núcleos para la penetración española en aquella región, con vistas, sin duda, a realizar en el futuro un camino rápido desde Quito a la costa.

El documento que va a servir de base para el estudio que viene a continuación, que ya había sido publicado previamente por Monroy, ${ }^{8}$ aunque con algunos errores de transcripción, se conserva en el Archivo General de Indias de Sevilla (legajo 1.077 de Documentos Escogidos). Habiendo sido nuevamente transcrito por la

6 Jijón y Caamaño, Jacinto: El Ecuador Interandino y Occidental antes de la conquista Castellana. 3 vols. Quito, 1941, vol. II. pág. 81.

7 Folio 1 del documento al que nos referimos por extenso en estas páginas.

8 Monroy: El Convento... Quito, 1958, págs. 313-366. 
doctora Sara Rodicio, nuestras referencias se harán siempre al documento original.

La descripción detallada de este documento es como sigue:

"Traslado del asiento que el año próximo pasado de noventa y siete el doctor Juan del Barrio de Sepúlveda oydor de la Real Audiencia de San Francisco de Quito (...) por comisión della, en nombre y por el Rey nuestro Señor toma con los caciques y curacas principales de la provincia de Lita (...). Va escrito todo en 27 hojas con ésta».

(1) “Asiento que el doctor Juan del Barrio de Sepúlveda oydor de Quito en nombre y por el Rey Nuestro Señor tomó con estos caciques para la nueva conversión a nuestra santa fee cathólica destos infieles y que sean vasallos de Su Magestad (...).. (Sábado 31 de mayo de 1597), fols. 1r. a Iv.

(2) "Título de Gobernador de los Yndios que se poblaren en lia provincia de Lita a don Alonso Gualapiango, caçique del pueblo de Lita). (Quito, 16 de junio de 1597), fols. 2r. a 3r.

(3) "Al corregidor de Otabalo que ayude a fray Gaspar de Torres y don Alonso Gualapiango para que vayan a los yndios que están adelante de Lita a lo que se les a ordenado y no consienta que vayan españoles a ello ni otras personas so color de descubrir minas ni camino». (Quito, 16 de junio de 1597), fols. 3v. a 4r.

(4) "Instrucción y orden que por mandado de la Real Audiencia y Chancillería que rreside en la çiudad de San Francisco de Quito, an de guardar don Luis Gualapiango y don Alonso Gualapiango, su hijo mayor, caçiques principales del pueblo de Lita que son de la Corona Real y el padre fray Gaspar de Torres de la orden de Nuestra Señora de las Merçedes (...)). (Quito, 16 de junio de 1597), fols. 4r. a 5v.

(5) "Memoria de lo que los dichos Padre Fray Gaspar de Torres y don Alonso Gualapiango, cacique y gobernador an hecho en virtud de las Reales Provisiones e ynstrucçión suso incorporadas que es como sigue». (20 de noviembre de 1597), fols. 8r. a 12v. 
(6) "Libro del Sancto bautismo de las Yglesias del Espíritu Santo y de Nuestra Señora Sancta María de Guadalupe de las provinģias de Singobucho, de Cayapa y Cunaha y Aguatene y Yambas y Lachas que yo fray Gaspar de Torres de la horden de nuestra Señora de las Merçedes, rredemptión de cautibos, e hechos con licencia del Perlado comendador fray Juan de Salas, de la casa de Quito y con liçençia de los señores Presidente y oydores que para ello me dieron, que al presente residen en la Real Audiencia de Quito, conforme a la Real Provisión que me dieron para que viniese acabar de hazer la redución de los yndios naturales destas dichas provinçias.

Que es fecho en diez y ocho dias del mes de Agosto del año de mill y quinientos y noventa y siete años. 1597", fols. 13r. a $26 \mathrm{r}$.

(7) "Carta del dicho Padre fray Gaspar de Torres (...) al Rey nuestro Señor". (5 de enero de 1598), fol. 26v.

(8) "Carta del padre fray Gaspar de Torres (...) al doctor Juan del Barrio de Sepúlveda). (9 de marzo de 15)8), fols. 27r a 27v.

El viaje de fray Gaspar de Torres por tierras de los cayapas, lachas, yambas y malabas duraría desde el 8 de julio hasta el 20 de noviembre de 1597 en que el fraile termina su Memorial. Durante esos 136 días se fundaron los pueblos «nuevos» de Espíritu Santo y Santa María de Guadalupe y se bautizaron 1.484 indios de los grupos ya mencionados.

El martes 8 de julio de 1597 los expedicionarios salen de Lita y, tras cuatro leguas de camino, llegan a pernoctar en el cerro Lonteotobucho. El segundo día fue mucho más duro que el primero, ya que no sólo caminaron una legua más, sino que el viaje se desarrolló por una zona sumamente quebrada, atravesando primero el riachuelo Lonteotopi y llegando a pernoctar junto a otro riachuelo: el llamado río Pitonpuno. Al día siguiente, jueves, los expedi- 
cionarios prosiguieron la marcha atravesando quebradas y arroyos hasta llegar al cerro de Malbucho o tierra de víboras, lo que representó una jornada de cuatro leguas de camino. El 11. de julio lo dedicaron los viajeros al descanso una vez que alcanzaron una chacra de maíz, propiedad de don Alonso Gualapiango.

El sábado 12 de julio se pusieron en marcha de nuevo los expedicionarios alcanzando a media legua de Malbucho el río de Tumpibucho. Una vez que atravesaron ese río «llegaron ocho yndios, naturales desta tierra, sujetos a don Diego Natinguila y - según dice Gaspar de Torres- nos trajeron de refresco en señal de amor, pescado coçido y tórtolas y bollos yamor, que por otro nombre se llama chicha» (fol. 8v.). No muy lejos, siguiendo el curso del río Tumpibucho se encontraron con Diego Natinguila, a quien el propio fray Gaspar de Torres había convertido al cristianismo anteriormente. Acompañados por el «principal» cayapa, llegaron a su casa a la caída de la tarde, en un lugar llamado Çunaha. Allí pasaron la noche y al día siguiente, domingo, 13 de julio de 1597, Gaspar de Torres mandó hacer una iglesia donde dijo misa. Allí iban a estar ocho días dedicados a la catequesis y el bautismo de los inclios; fue entonces cuando parte de los indios que acompañaban a los españoles - probablemente pastos o quillacingas - regresaron a Lita, de donde procedían.

El 21 de julio, el cacique Francisco Cayapa condujo a fray Gaspar de Torres hasta su pueblo de Singobucho, distante unas cuatro leguas de Çunaha. En Singobucho el cacique mandó construir una iglesia y la casa del fraile, haciéndose construir otra casa para él mismo. El martes, 22 de julio, fray Gaspar bendijo la iglesia que dedicó al Espíritu Santo y celebró su primera misa. Desde ese día al 26 de septiembre el misionero residió en el lugar dedicándose a predicar, catequizar y bautizar a los numerosos indios cayapas de la zona.

El 26 de septiembre de 1597 fray Gaspar de Torres y sus acompañantes dejaron la casa de Francisco Cayapa en Singobucho y se dirigieron hacia Aguatene, viaje en el que emplearon dos días, atravesando numerosos cursos fluviales, entre los cuales, los ríos 
Cupi, Lachapi, Picholipi, Silamboyo y Lenguepi, llegando finalmente a la casa del principal Aguazami, quien ya había mandado construir una iglesia en el pueblo de Aguatene. Esa iglesia que iba a dedicar a Santa María de Guadalupe sería bendecida el día 28 de septiembre de 1597. Fray Gaspar iba a residir en aquel pueblo los siguientes 24 días predicando y bautizando a los indios de la región.

El miércoles, 22 de octubre, la expedición misionera parte de Aguatene, dirigiéndose hacia tierras de lachas y yambas, región situada a cuatro leguas de aquel pueblo y en la que en 1595 ya había bautizado a numerosos indios. El domingo siguiente, 26 de octubre, llegaron al pueblo de Çaraha, donde se había construido una iglesia que fray Gaspar iba a dedicar a Santa María de Guadalupe y donde celebró misa y predicó desde aquel día, bautizando a numerosos indios.

Como ya dijimos, el Memorial de fray Gaspar de Torres finaliza el día 20 de noviembre; sin embargo, en el Libro del Sancto bautismo que se reproduce a continuación del Memorial se copian las actas de bautismos correspondientes a la expedición verificada por aquella zona en los meses de junio a agosto de 1595.

\section{Pueblos y GRUPOS Ét'TICOS}

Como vamos a ver con detalle en las páginas siguientes el análisis de los nombres indígenas que se registran en las listas de bautismos de fray Gaspar de Torres, en el documento que nos sirve de base para este estudio, nos permitirá precisar las áreas o subáreas lingüísticas dentro de la región, en especial las relativas a lachas, yambas y malabas, que son aquellas a las que el padre Torres describió peor en su Memorial, por no haberlas recorrido en el año 1597, o porque, correspondiendo a la última parte de su expedición, prestó menor atención. En cualquier caso, en la tabla siguiente (Tabla 1) relacionamos abreviadamente los datos aportados por el manuscrito, de modo que nos sirva de base para la investigación posterior. 


\section{TABLA 1}

Fecha Localidad Etnia Cacique fiolio

\begin{tabular}{|c|c|c|c|c|}
\hline 20-VIII-97 & Singobucho & Cayapas & Francisco Cayapa & $14 \mathrm{r}$ \\
\hline $30 \cdot V 111-97$ & Singobucho & Cayapas & Francisco Cayapa & \\
\hline 5-IX-97 & Singobucho & Cayapas & Francisco Cayapa & \\
\hline 8-IX-97 & Singobucho & Cayapas & Francisco Cayapa & \\
\hline 10-IX-97 & Singobucho & Cayapas & Francisco Cayapa & \\
\hline 12-IX.97 & Singobucho & Cayapas & Francisco Cayapa & \\
\hline 14-IX.97 & Singobucho & Cayapas & Gaspar Uñatapa & \\
\hline 16-IX-97 & Çunaha & Cayapas & Diego Natinguila & \\
\hline 18-IX-97 & Çunaha & Cayapas & Diego Natinguila & \\
\hline 20-IX-97 & Moesilli & Cayapas & Pedro Chilmisso & \\
\hline 22-IX.97 & Moesilli & Cayapas & Pedro Chilmisso & \\
\hline 24-IX.97 & & Cayapas & Juan Pifiqui & \\
\hline 5-X-97 & Aguatene & Cayapas & Juan Aguazami & \\
\hline 6-X-97 & Aguatene & Cayapas & Juan Yahatino & \\
\hline 12-X-97 & Aguatene & Cayapas & Diego Zami & \\
\hline 28-X-97 & Çaraha & Yambas & Martín Nagola & \\
\hline 2.XI.97 & Çaraha & Yambas & Martín Nagola & \\
\hline 4.XI-97 & Çaraha & Yambas & Juan Tapiba & \\
\hline 5-XI-97 & & Pillalaguas & Diego Oñapapa & \\
\hline 6-XI-97 & & Malabas & Garçía Canbila & \\
\hline 6-VIII-95 & & Yambas & Diego Pisopuchopuele & \\
\hline \multirow[t]{2}{*}{ 11.VIII-95 } & Calaxo & & Juan Tapiba & \\
\hline & Pisopuchopuele & & Juan Mayba & \\
\hline 15-VIII-95 & & & Diego Aguapapa & \\
\hline \multirow[t]{3}{*}{ 16-VIII-95 } & Pillalagua & Lachas & Sancho Aguatata & \\
\hline & Çaraha & & Martín Nagola & \\
\hline & Çaraha & & Martín Nagola & \\
\hline $1 . V I .95$ & & Yambas & Juan Tapiba & \\
\hline $1 . V I .95$ & & & Felipe Canhiba & \\
\hline
\end{tabular}

De la relación anterior se desprende que los grupos étnicos de la zona que estudiamos son los cayapas, yambas, pillalaguas, malabas y lachas (véase Mapa 1). El posterior análisis de las series 
nominales nos permitirán apreciar hasta qué punto se pueden mantener esos grupos con sus respectivos nombres o no.

Sin embargo, como paso previo al anunciado análisis de las series nominales trataremos de dos aspectos que consideramos fundamentales: los ayllus y su demografía.

Con respecto al concepto de ayllu que repetidamente emplea fray Gaspar de Torres en sus listas de bautizados, ya hemos dicho en ocasión anterior ${ }^{9}$ que ese término es quechua $\mathrm{y}$ debe ser interpretado en el contexto que estudiamos como «grupo étnico» y no en la forma en que literalmente se utilizaba en el período de dominación inca de las Andes septentrionales, ya que la región a la que nos estamos refiriendo nunca formó parte del Tawantinsuyu.

Según hemos dicho en otro lugar, «la unidad básica de toda la organización social incaica, por encima de la familia, era el ayllu, el cual venía a ser como una extensión de la familia, o una subdivisión de la tribu. De esta agrupación se han dado numerosas definiciones. Para Valcárcel es básicamente una unidad económica caracterizada por el control de tierras y trabajo. Según Rowe, el ayllu inca era un grupo de parentesco con endogamia teórica, con descendencia por línea masculina. Kirchhoff dice del ayllu que es un grupo permanentemente basado en una descendencia común real o supuesta de sus miembros, mientras Baudin afirma que estaba formado por todos los descendientes de un real o supuesto antecesor. Esencialmente pues, el ayllu representaba una unidad de parentesco en la que los miembros se consideraban descendientes de un antepasado común. ${ }^{10}$

La interpretación que estamos dando a los grupos a los que designa fray Gaspar de Torres como ayllus es, por lo tanto, semejante: grupos de individuos que, por encima de la familia, se consideraban emparentados y, por lo tanto, serían familias conyugales que se reconocerían descendientes de un antepasado común.

Cada uno de esos ayllus queda definido por el nombre de un

9 Palop: Los cayapas... (iuayaquil, 1986, pág. ¿37.

10 Alcina, José y Palop, Josefina: Los incas. En «llistoria de América, I: Prehistoria e Historia Antigua», págs. 413-476. Madrid, 1987, pág. 431. 
cacique, jefe o principal, que podría ser simplemente el parientc mayor o más representativo desde el punto de vista social, pero que también podría acumular algún género de poder político, a juzgar por las expresiones de fray Gaspar. Así, cuando, al referitse al ayllu de Juan Nagola, dice:

"...en este asiento de (aaraha, como arriba digo, esta un principal llamado don Martín Nagola, con número de yndios a él subjetos...) (fol. 22r.).

La serie de ayllus referidos en el documento que estudiamos son 21 , según la relación siguiente (Tabla 2).

TABLA 2

\begin{tabular}{|c|c|c|c|}
\hline$N . "$ & Jefe de ayllu & Fechas bautismo & fiolios \\
\hline 1 & Francisco Cayapa & $20 . \mathrm{V} 111$ a $12.1 \mathrm{X}-97$ & $14 \mathrm{r} .-16 \mathrm{v}$. \\
\hline 2 & Gaspar Uñatapa & $14-1 X-97$ & $17 \mathrm{r}$ \\
\hline 3 & Diego Natinguila & $16-18-1 X-97$ & $17 \mathrm{v} .-18 \mathrm{v}$ \\
\hline 4 & Pedro Chilmisso & $20 \cdot 22 \cdot \mathrm{IX} \cdot 97$ & $18 \mathrm{v} .19 \mathrm{v}$. \\
\hline 5 & Juan Pifiqui & 24.-1X-97 & $19 \mathrm{v}$ \\
\hline 6 & Juan Aguazami & $5 \cdot X \cdot 97$ & $20 \mathrm{r}$. \\
\hline 7 & Juan Yahatino & 6.X.97 & $21 r$. \\
\hline 8 & Diego Zami & $12 \cdot X \cdot 97$ & $21 \mathrm{v}$ \\
\hline 9 & Martín Nagola & $28 . X$ a $2 \cdot X 1.97$ & $22 \mathrm{r} \cdot 2 \cdot 2 \mathrm{v}$ \\
\hline 10 & Juan Tapiba & 4.XI-97 & $23 r$ \\
\hline 11 & Diego Oñapapa & 5.XI-97 & $23 r$ \\
\hline 12 & Garçía Canbila & $6 \cdot X I \cdot 97$ & $23 \mathrm{v}$. \\
\hline 13 & Diego Supuchopuele & 6.VIII.95 & $23 v$. \\
\hline 14 & Juan Tapiba & 11.VIII.95 (?) & 24 r. \\
\hline 15 & Juan Mayba & & $24 \mathrm{v}$. \\
\hline 16 & Diego Aguapapa & 15.VIII-95 & $24 \mathrm{v}$. \\
\hline 17 & Sancho Aguatata & $10 \cdot$ VIII.95 & $25 r$. \\
\hline 18 & Martín Nagola & & $25 \mathrm{v}$. \\
\hline 19 & & & $25 \mathrm{v}$ \\
\hline 20 & Juan Tapiba & $1 \cdot V I-95$ & $25 \mathrm{v} .26 \mathrm{r}$. \\
\hline 21 & Felipe Canbiba & 1.VI.95 & $26 r$. \\
\hline
\end{tabular}


Los cinco primeros ayllus parecen corresponder al grupo étnico cayapa cuya cabecera se hallaba en Singobucho y cuyo cacique principal era Francisco Cayapa quien en 1587, ayudado por los caciques Luis y Alonso Gualapiango había abandonado el pueblo de Campi o Çampi a causa de los continuados ataques de negros y mulatos de Esmeraldas, instalándose entonces en Singobucho. El hecho de que los principales Gaspar Uñatapa, Diego Natinguila, Pedro Chilmisso y Juan Pifique, fuesen parientes y jefes de ayllus, permite suponer que el grupo cayapa desde el punto de vista de la organización política, podría interpretarse como un grupo tribal complejo o como una jefatura extremadamente simple.

Todos los demás ayllus, según veremos en las páginas siguientes, deberán agruparse de manera diferente a la secuencia presentada por el misionero. Es importante, sin embargo, que examinemos en este momento los datos de carácter demográfico de que disponemos para estas poblaciones.

Utilizando las listas de bautismos de cada uno de los ayllus, obtendríamos el siguiente resultado (vid. Tabla 3):

TABLA 3

Ayllu Nombre del cacique

Mujeres

Hombres

Totales

1 Francisco Cayapa

137

33

301

438

2 Gaspar Uñatapa

40

58

91

3 Diego Natinguila

4.2

74

114

4. Pedro Chilmisso

90

132

5 Juan Pifiqui

15

16

6 Juan Aguazami

1

72

138

7 Juan Yahatino

66

50

93

8 Diego Zami

30

48

9 Martín Nagola

18

41

73

10 Juan Tapiba

32

11

33

44

II Diego Oñapapa

3

15

18

12 Garçía Canbila

1

7

8

T'omo XLVII 


$\begin{array}{llccc}\text { Ayllu Nombre del cacique } & \text { Mujeres } & \text { Hombres } & \text { Totale's } \\ & & & \\ 13 & \text { Diego Supuchopuele } & 1 & 23 & 24 \\ 14 & \text { Juan Tapiba } & 23 & 29 & 52 \\ 15 & \text { Juan Mayba } & 15 & 15 & 30 \\ 16 & \text { Diego Aguapapa } & 17 & 26 & 4.3 \\ 17 \text { Sancho Aguatata } & 22 & 36 & 58 \\ 18 \text { Martín Nagola } & 16 & 20 & 36 \\ 19 & & 2 & 2 \\ 20 & \text { Juan Tapiba } & 1 & 11 & 12 \\ 21 & \text { Felipe Canbiba } & & 14 & 14\end{array}$

$$
\begin{array}{llll}
\text { Totales } & 522 & 102 & 1.411
\end{array}
$$

Si comparamos esos datos con el resumen que el propio frily Gaspar de Torres proporciona en el documento que estudiamos (fol. 26r.) y que reproducimos a continuación, observaremos escasas diferencias.

\section{TABLA 4}

Cayapa Craraha T'otules

\begin{tabular}{|c|c|c|c|}
\hline Hombres & 340 & 265 & $0(0.5$ \\
\hline Mujeres & 179 & 173 & 3.52 \\
\hline Muchachos & 193 & 131 & 321 \\
\hline Muchachas & 69 & 93 & 102 \\
\hline
\end{tabular}

Si comparamos las cifras dadas para cayapas en una y otra tablit observaremos que la diferencia no es, en conjunto, de más de diéz individuos a favor de las cifras registradas en las listas de bautismos: hombres y muchachos son 533 y en las listas, 538, mientras las mujeres y muchachas son 248 y en las listas suman 253. 
Aunque la diferencia entre hombres y muchachos y mujeres y muchachas ni es precisa, ni corresponde a una misma edad, ya que la madurez masculina y femenina no es nunca coincidente, la matización del resumen dado por fray Gaspar de Torres, completa favorablemente los datos de las listas.

Hay que destacar, no obstante, que la propia cifra global de 1.443 ó 1.484 individuos parece muy baja para el territorio total de que estamos tratando. Ello queda explicado por el propio fray Gaspar de Torres cuando dice que esas cifras son «sin los que faltan por bautizar» que serían, sin duda, bastantes. Sería muy arriesgado tratar de calcular esa población sin bautizar, por lo que deberemos admitir simplemente esas cifras como un dato de difícil valoración.

Es mucho más notable e irregular la diferencia entre hombres y mujeres que si en muchos ayllus viene a ser aproximadamente la mitad de mujeres respecto a los hombres, en otros casos llega a ser casi inexistente. Eso es lo que sucede en los ayllus 5, 11, 12, 13, 19,20 y 21 , donde apenas se consignan nombres de mujer que hayan sido bautizadas. Por el contrario, en algunos ayllus, las mujeres casi llegan a igualar a los hombres; ese es el caso de los ayllus $6,7,14$ y 15. La explicación de estas desigualdades sólo puede hallarse en el terreno de las conjeturas más o menos lógicas como puede ser la del infanticidio de niñas, negativa de las mujeres a ser bautizadas a causa de su mayor timidez, etc.

\section{ANÁLISIS DE LAS LISTAS DE BAUTISMOS}

Del análisis de los nombres propios de los indios que fueron bautizados por fray Gaspar de Torres, se distinguen con toda claridad dos grupos: el de los cayapas (ayllus $1,2,3,4$ y 5) y el de los malabas (ayllus 10, 12, 13, 14, 15, 19, 20 y 21). Los restantes ayllus podrían ser calificados de mestizos, en tanto que en todos cllos apreciamos la presencia de individuos - hombres y mujerescon nombres cayapas al tiempo que se aprecian otros individuos 
con nombres diferentes: los ayllus 6, 7, 8, 9 y 11 presentan una lista de nombres nunca repetidos y exclusivamente masculinos que podrían corresponder a lachas y/o yambas; por su parte, en los ayllus 16,17 y 18 aparecen otros nombres - distintos a los anteriores- propios de hombres, pero también algunos de mujeres que por su localización podrían atribuirse a los llamados pillalaguas. Veamos ahora cada uno de esos grupos.

\section{El GRUPO CAYAPA}

Las listas de nombres de hombres y mujeres bautizados por fray Gaspar de Torres, de la etnia cayapa comprende los ayllus 1 a 5 que son los propiamente cayapas, según hemos visto más arriba y los nombres de cayapas que aparecen en los ayllus 6 a 9, 11 y 16 a 18. Este conjunto de nombres que han sido recogidos en las Tablas 5 (hombres) y 6 (mujeres) incluyen 45 nombres de mujer y 69 nombres de hombre.

Los nombres más frecuentemente representados en las listas son los siguientes:

a) Hombres: 1/Aguapapa (34).

2/Anapira (73).

3/Añapa (88).

4/Atapa (60).

5/Napapa (106).

6/Uñapapa u Oñapapa (145).

b) Mujeres: 1/Aguamama (46).

2/Añama (56).

3/Namama (94).

4/Uñamama u Oñamama (122). 
Estos diez nombres representan el $75 \%$ de los nombres de hombres y el $69 \%$ del de las mujeres. El sufijo de los nombres personales designan de manera casi universal el sexo de la persona ya que ma para las mujeres y pa para los hombres son constantes en ambas listas.

De los varios estudios sobre el parentesco cayapa en la actualidad, tales como los de Barrett, "Jijón ${ }^{12}$ o Carrasco ${ }^{13}$ se pueden extraer una serie de términos que servirían para interpretar los nombres propios cayapas.

$$
\begin{aligned}
\text { apa } & =\text { padre } \\
\text { papa } & =\text { padre } \\
\text { yapa } & =\text { padre } \\
\text { na } & =\text { hijo } \\
\text { naa } & =\text { hijo } \\
\text { na } & =\text { niño } \\
\text { paña } & =\text { nieto }
\end{aligned}
$$

$$
\begin{aligned}
\text { ama } & =\text { madre } \\
\text { mama } & =\text { madre } \\
\text { yana } & =\text { madre } \\
\text { nama } & =\text { hija } \\
\text { apamama } & =\text { abuela }
\end{aligned}
$$

El hecho de que varios de esos términos aparezcan en los nombres propios de los indios, ofrece una apariencia en éstos como si verdaderamente expresasen los tipos de parentesco. La presentación de varias de las familias con un mayor número de miembros en varias generaciones nos demostrará hasta qué punto esa apreciación es totalmente engañosa.

La familia de Francisco Cayapa, el cacique de esa posible jefatura cayapa y el que da nombre a todo el grupo en la época colonial, puede ser el mejor ejemplo sobre lo que estamos didiendo:

11 Barret. S. A.: The Cayapa indians of Ecuador. Indian Noles and Mono graphs, núm. 40. Museum of the American Indian. Heye Foundation, 2 vols. New York, 1925.

12 Jijón: El Ecuador interandino...

13 Carrasco, Eulalia: El pueblo Chachi. El jeenoume avanza. Quito, 1983. 


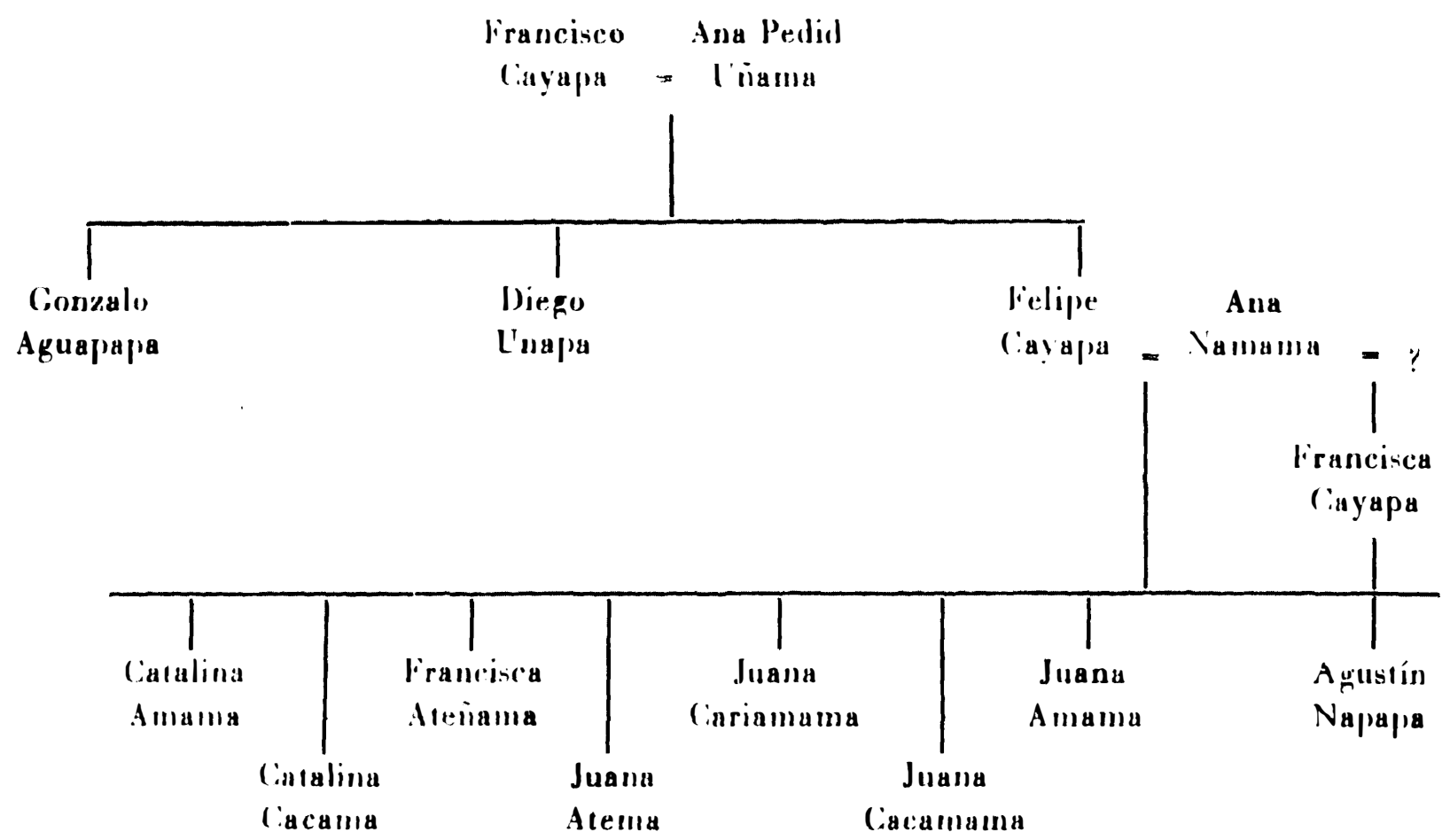

Otras familias conyugales cayapas que pueden servir de ejemplo son las siguientes:
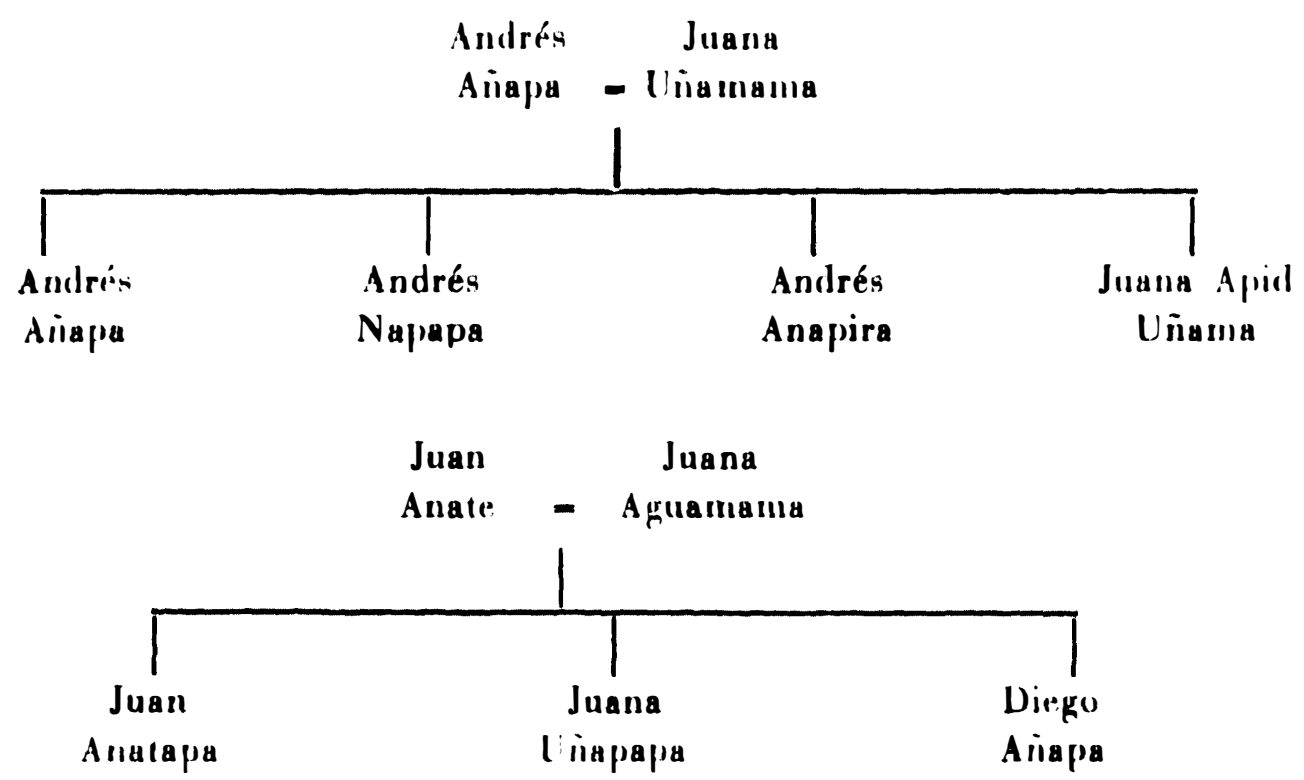
No obstante, lo que es evidente es que la región o territorio estrictamente cayapa la concentración de nombres con las terminaciones típicas de los cayapas es muy alta, mientras que en áreas en que los cayapas conviven con otras etnias los nombres dominantes son escasos: Aguapapa, Carapapa, Napapa, Uñapapa, Namama y Uñamama u Oñapapa.

\section{TABLA 5}

Nombres cayapas: hombres

\begin{tabular}{|c|c|c|c|c|c|c|c|c|c|c|c|c|}
\hline \multirow[b]{2}{*}{ Nombres } & \multirow[b]{2}{*}{1} & \multirow{2}{*}{2} & \multirow[b]{2}{*}{3} & \multirow[b]{2}{*}{4} & \multicolumn{2}{|c|}{ Ayllus } & \multirow[b]{2}{*}{7} & \multirow[b]{2}{*}{8} & \multirow[b]{2}{*}{$9 \quad 11$} & \multirow[b]{2}{*}{16} & \multirow{2}{*}{$17 \quad 1$} & \multirow[b]{2}{*}{ Total } \\
\hline & & & & & 5 & 6 & & & & & & \\
\hline Abencolu & 1 & & & & & & & & & & & 1 \\
\hline Abunago & 1 & & & & & & & & & & & $\begin{array}{lll}1 & & l \\
\end{array}$ \\
\hline Acapa & 1 & & & & & & & & & & & 1 \\
\hline Achuapa & & & 1 & & & & & & & & & 1 \\
\hline Aguagua & & & & 2 & & & & & & & & 2 \\
\hline Aguapapa & 4 & 1 & 5 & 2 & & 6 & 1 & 2 & 4 & 5 & 4 & 34 \\
\hline Ahuemo & 1 & & & & & & & & & & & 1 \\
\hline Anapa & 6 & 2 & & & & & & & & & & 8 \\
\hline Anapira & 45 & 5 & 14 & 8 & 1 & & & & & & & 73 \\
\hline Anata & 1 & & & & & & & & & & & 1 \\
\hline Anatapa & 11 & 2 & & & & & & & & & & 13 \\
\hline Anate & 11 & 3 & 2 & 2 & & & & & & & & 18 \\
\hline Anati & 1 & & & & & & & & & & & 1 \\
\hline Anatiapa & 1 & & & & & & & & & & & 1 \\
\hline Añaca & & & & 1 & & & & & & & & 1 \\
\hline Añapa & 50 & 9 & 5 & 21 & 3 & & & & & & & 88 \\
\hline Apapa & 1 & & & 2 & 1 & & & & & & & 4 \\
\hline Apiduñapa & 2 & & 1 & & & & & & & & & 3 \\
\hline Apira & 6 & & 3 & 5 & & & & & & & & 14 \\
\hline Apirara & 2 & & 1 & 1 & & & & & & & & 4 \\
\hline Atapa & 39 & 8 & 2 & 7 & 4 & & & & & & & 60 \\
\hline Atapapa & & & & 1 & & & & & & & & 1 \\
\hline Atape & & & 1 & & & & & & & & & 1 \\
\hline Atipapa & 1 & & & & & & & & & & & 1 \\
\hline
\end{tabular}

6. Anuario 
Nombres cayapas: hombres (continuación)

\begin{tabular}{|c|c|c|c|c|c|c|c|c|c|c|c|c|c|c|}
\hline & & & & & & llus & & & & & & & & \\
\hline Nombres & 1 & 2 & 3 & 4 & 5 & 6 & 7 & 8 & 9 & 11 & 16 & 17 & 18 & T'otal \\
\hline Cadmiso & & & & 1 & & & & & & & & & & 1 \\
\hline Cacapa & 1 & & & & & & & & & & & & & l \\
\hline Cala & & & & & & & & 1 & & & & & & ] \\
\hline Calala & 7 & 2 & & 2 & & & & & & & & & & 11 \\
\hline Canoui & & 1 & & 1 & & & & & & & & & & 2 \\
\hline Carapapa & 2 & & & & & 1 & & & 1 & & 1 & 2 & & 7 \\
\hline Cariapa & 1 & 1 & & & & 1 & & & & & & & & 3 \\
\hline Cariapapa & 2 & 1 & 2 & 1 & & & 2 & & & 1 & & & & 9 \\
\hline Cauapapa & & & & & & 1 & & & & & & & & 1 \\
\hline Cayapa & 2 & & & & & & & & & & & & & 2 \\
\hline Caga & 1 & & & & & & & & & & & & & 1 \\
\hline Çayabue & 1 & & & & & & & & & & & & & 1 \\
\hline Cequi & 1 & & & & & & & & & & & & & 1 \\
\hline Chilmisso & & & & 1 & & & & & & & & & & 1 \\
\hline Çuçu & 1 & & & & & & & & & & & & & 1 \\
\hline Çucho & 1 & & & & & & & & & & & & & 1 \\
\hline Cungu & & & & 1 & & & & & & & & & & 1 \\
\hline Curro & l & & & & & & & & & & & & & 1 \\
\hline Hucumapa & 1 & & & & & & & & & & & & & 1 \\
\hline Hucumo & 3 & & & & & & & & & & & & & 3 \\
\hline Lahaque & 1 & & & & & & & & & & & & & 1 \\
\hline Laque & 3 & & & & & & & & & & & & & 3 \\
\hline Mandinchi & & & 1 & & & & & & & & & & & 1 \\
\hline Mugoapapa & & & & & & & & 1 & & & & & & l \\
\hline Naapa & l & & & & & & & & & & & & & ] \\
\hline Napa & & 1 & & & & & & & & & & & & 1 \\
\hline Napapa & 34 & 9 & 17 & 14 & 2 & 13 & 4 & 5 & 4 & 1 & 2 & l & 1 & 107 \\
\hline Naçago & 3 & & & & & & & & & & & & & 3 \\
\hline Natapa & & & & 1 & & & & & & & & & & 1 \\
\hline Natinguila & & & 1 & & & & & & & & & & & 1 \\
\hline Ocuma & 1 & & & & & & & & & & & & & 1 \\
\hline Pifiqui & & & & & 2 & & & & & & & & & 2 \\
\hline
\end{tabular}


Nombres cayapas: hombres (continuación)

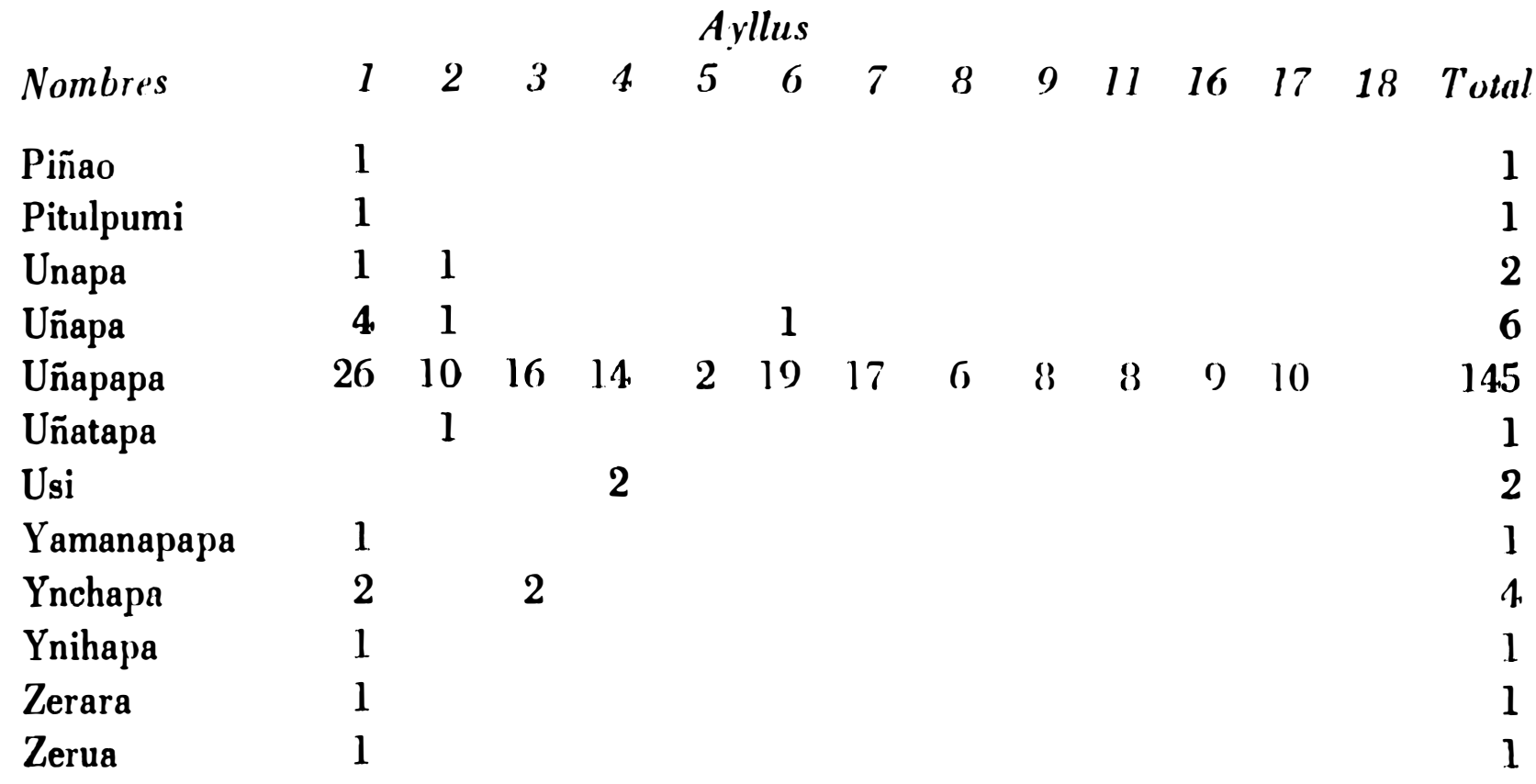

$\begin{array}{lllllllllllllll}\text { TOTAIES } & 292 & 58 & 74 & 90 & 15 & 42 & 24 & 15 & 17 & 10 & 17 & 17 & 1 & 672\end{array}$

TABLA 6

Nombres cayapas: mujeres

\begin{tabular}{|c|c|c|c|c|c|c|c|c|c|c|c|c|c|}
\hline \multicolumn{14}{|c|}{ Ayllıs } \\
\hline Nombres & 1 & 2 & 3 & 4 & 50 & 7 & 8 & 9 & 11 & 16 & 17 & 18 & Total \\
\hline Aguamama & 4 & 1 & 5 & 1 & 15 & 7 & 3 & 6 & 1 & 1 & 1 & 1 & 46 \\
\hline Amama & 2 & 1 & & & 2 & & & & & & & & 5 \\
\hline Amia & 2 & & & & & & & & & & & & 2 \\
\hline Anama & 3 & & & & & & & & & & & & 3 \\
\hline Anamama & 1 & & & & & & & & & & & & 1 \\
\hline Anañama & 1 & & & & & & & & & & & & 1 \\
\hline Añama & 30 & 7 & 5 & 13 & & & 1 & & & & & & 56 \\
\hline Añamama & 7 & 1 & & 2 & & & 1 & & & & & & 11 \\
\hline Aomama & & & 1 & & & & & & & & & & 1 \\
\hline Apiduñama & 6 & 2 & & & & & & & & & & & 8 \\
\hline Apiquña & 1 & & & & & & & & & & & & 1 \\
\hline Atema & 1 & & & & & & & & & & & & \\
\hline
\end{tabular}




\section{Nombres cayapas: mujeres (continuación)}

\begin{tabular}{|c|c|c|c|c|c|c|c|c|c|c|c|c|c|c|}
\hline & & & & & & Illus & & & & & & & & \\
\hline Nombres & 1 & 2 & 3 & 4 & 5 & 6 & 7 & 8 & 9 & 11 & 16 & 17 & 1ii & T'otal \\
\hline Atenama & 4 & 1 & & & & & & & & & & & & 5 \\
\hline Ateñama & 3 & 3 & 1 & 1 & & & & & & & & & & 8 \\
\hline Atinama & 3 & & & & & & & & & & & & & 3 \\
\hline Atinuñama & l & & & & & & & & & & & & & 1 \\
\hline Atiñama & 3 & 1 & & & & 1 & & & & & & & & 5 \\
\hline Cacama & 1 & & & & & & & & & & & & & 1 \\
\hline Caçama & & 1 & 1 & & & & & & & & & & & 2 \\
\hline Cacamama & 1 & & & & & & & & & & & & & 1 \\
\hline Caçamama & & & 1 & & & & & 1 & & & & & & 2 \\
\hline Calala & 1 & & & & & & & & & & & & & 1 \\
\hline Calamama & 2 & 2 & 1 & 3 & & l & & 1 & & & & & & 10 \\
\hline Caramama & 2 & & & & & l & & & & & & 1 & & 4 \\
\hline Cariamama & 6 & & 4 & 1 & & 3 & 1 & & & & & 1 & & 16 \\
\hline Cauamama & & & & & & 1 & & & & & & & & 1 \\
\hline Cayapa & 1 & & & & & & & & & & & & & 1 \\
\hline Cumama & 1 & & & & & & & & & & & & & 1 \\
\hline Çumama & 1 & & & & & & & & & & & & & 1 \\
\hline Humama & 2 & & & & & & & & & & & & & 2 \\
\hline Mogonamama & & & & & & & & 1 & & & & & & 1 \\
\hline Naamama & & & 2 & & & & & & & & 4 & 6 & 2 & 14 \\
\hline Namama & 12 & 7 & 7 & 11 & 1 & 18 & 19 & 5 & 9) & 1 & & 2 & 2 & 0.1 \\
\hline Nañama & 5 & 1 & 1 & 4 & & & & & & & & & & 11 \\
\hline Naoñamama & & & & & & & 1 & 1 & & & & & & 2 \\
\hline Naumama & 1 & & & & & & & & & & & & & 1 \\
\hline Namama & 1 & & 1 & & & 1 & & & & & & & & 3 \\
\hline Oñama & & & & & & 1 & & & 1 & & & & & 2 \\
\hline Oñamama & & & & & & 22 & 15 & 4 & 16 & & 12 & 6 & 3 & 78 \\
\hline Pillama & 1 & & & & & & & & & & & & & 1 \\
\hline Unamama & 1 & & & & & & & & & & & & & 1 \\
\hline Uñamama & 24 & 5 & 10 & 5 & & & & & & & & & & 44 \\
\hline Ynchama & & & & 1 & & & & & & & & & & 1 \\
\hline TOTALES & 125 & 33 & 40 & 42 & 1 & 56 & 43 & 18 & 32 & 2 & 17 & 17 & 9 & 455 \\
\hline
\end{tabular}


El grupo Malaba

De los malabas ${ }^{14}$ sabemos sobre todo que era un pueblo muy agresivo, que habitaba en las tierras bajas al sur de los ríos Mira y Mataje. A partir del análisis de los nombres de las listas de bautismos, podemos agrupar con los llamados malabas por fray Gaspar de Torres, es decir, el ayllu de Garçía Canbila, otra serie de ayllus situados algo más al interior, en territorio de Yambas, especialmente en la región de Calaxo y Pisopuchopuele, en la margen derecha del río Mira, en las primeras estribaciones de los Andes. Los ayllus 10,14 y 20 presentan un único principal o cacique, o varios con el mismo nombre, Juan Tapiba, mientras los restantes ayllus 13,15 y 21 tienen caciques cuyos nombres son típicamente malabas: Diego Supuchopuele, Juan Mayba y Felipe Canbiba.

Aunque es muy poco lo que se sabe de la lengua malaba, podemos afirmar que los nombres personales de hombre terminan casi sistemáticamente en $b a$ o iba (ver Tabla 7); no así los nombres de mujer, para los que las terminaciones más frecuentes son las de $c a$ o ica (Tabla 8).

Los nombres más comunes entre los ayllus malabas son los siguientes:

bombres

Caçiba

Pulaçiba o Pulasiba

Naciba o Naçiba

Tocoro

Tapiba. mujeres

Achica

Nachical

El hecho de que la suma de la población correspondiente a estos ocho ayllus no sobrepase los 186 individuos, de los que solamente 52 eran mujeres, prueba que estos grupos acudían al centro

14 Jijón: El Ecuador interandino..., pág. 540 y Palop: Los cayapas..., páginas 243-244. .

Tomo XLVII 
misional con muchas reservas y, por lo tanto, ese número de habitantes no puede considerarse representativo del conjunto del grupo.

Hay que advertir que los ayllus 14 y 15 de Juan Tapiba y Juan Mayba contienen dos mujeres cayapas, llamadas en el primer caso Uñamama y en el segundo Oñamama. Otro tanto podemos decir de Nagapapa, un hombre que se incluye en la lista del ayllu 14 de Juan Tapiba.

\section{TABLA 7}

Nombres malabas: hombres

\begin{tabular}{|c|c|c|c|c|c|c|c|c|c|}
\hline Nombres & 10 & 12 & 13 & 14 & 15 & 19 & 20 & 21 & T'otal \\
\hline Achecuyba & & & & 1 & & & & 1 & 1 \\
\hline Achiba & & & & & & & & 1 & 1 \\
\hline Achicoyba & & & & 2 & & & & & 2 \\
\hline Achicoyba & & & & & 1 & & & & 1. \\
\hline Acicuiba & & & & & & 1 & & & 1 \\
\hline Acicuymba & & & & & & & 1 & & 1 \\
\hline Acigoyla & & & & 2 & & & & & 2 \\
\hline Anguiba & & & & 2 & & & & & 2 \\
\hline Ayba & & & & 1 & & & & & 1 \\
\hline Azicuiba & & & & & & & & 1 & 1 \\
\hline Caciha & & & & & & 2 & & & 2 \\
\hline Caçiba & & & & 1 & 5 & & 1 & 2 & 9 \\
\hline Cambiba & 1 & & 2 & & & & & & 3 \\
\hline Campa & 1 & & & & & & & & 1 \\
\hline Canbiba & & & & 3 & & & & 2 & 5 \\
\hline Canbila & & 1 & & & & & & & 1 \\
\hline Cauba & & & 1 & & & & & & 1 \\
\hline Caziba & 1 & & & & & & & & 1 \\
\hline Cobola & 1 & & & & & & & & 1 \\
\hline Cuenbiba & & & & & 2 & & & & 2 \\
\hline Galliga & & & & & & & & l & 1 \\
\hline Gaziba & & & 1 & & & & & & 1 \\
\hline Hazicuyba & 1 & & & & & & & & 1 \\
\hline
\end{tabular}


nombres malabas: hombres (continuación)

Nombres

Lanba

Liba

Machiba

Magoche

Maquaziba

Mayba

Maynba

Naciba

Nachiba

Naçugiba

Nagapapa

Nanacuro

Napa

Nasiba

Natacoro

Naxiba

Nayba

Pasquiba

Polanba

Pulaçiba

Pulasiba

Pulasila

Pulaziba

Quasiba

Quiliba

Sallban

Supucho Puele

Tacoro

T'apiba

Zacoro
Maxiba

$\begin{array}{lllllllll}10 & 12 & 13 & 14 & 15 & 19 & 20 & 21 & \text { Total }\end{array}$

1

1

1

1

13

1

1

1

1

$1 \quad 1$

1

\section{1}

1

1

1

4

1

2

2

1

1

11

1

12

4

1

11

$1 \quad 1$

2

4

$11 \quad 1$

1

1

1

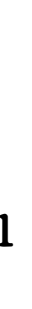

1

$\begin{array}{llll}3 & 3 & 4 & 1\end{array}$

$2 \quad 13$

$\begin{array}{lllll}5 & 1 & 3 & 4 & 1\end{array}$

14

1

$\begin{array}{ll} & 1 \\ 2 & 1 \\ 2 & 2\end{array}$

5

28

12

1

11

1

1

1

1

3
1

$\begin{array}{llllllllll}\text { TOTALES } & 33 & 7 & 23 & 29 & 15 & 2 & 10 & 14 & 133\end{array}$

Tomo XLVII 
TABLA 8

Nombres malabas: mujeres

Nombres

$10 \quad 12$

$$
13 \quad 14
$$

\section{5}

19

20

21 Total

Achi

Achica

Ascaquasi

$\begin{array}{lll}3 & 3 & 3 \\ & & 3 \\ & 1 & \end{array}$

1

1

Cacuy

Campechica

1

Canchiqui

Caui

Cobola

Cobulo

Cuachisca

1

Guachi

Hazcura

Huasibisca

1

1

Lavechica

Machica

Macua

Maguachiqui

Maquachi

Maqual

Mubisa

1

1

9

1

Nachica

Nacigal

Nahachica

Nascal

Nascay

Nasigua

Natacura

Natabuchica

Nazesica

1

1

1

2

1

1

1

1

1 


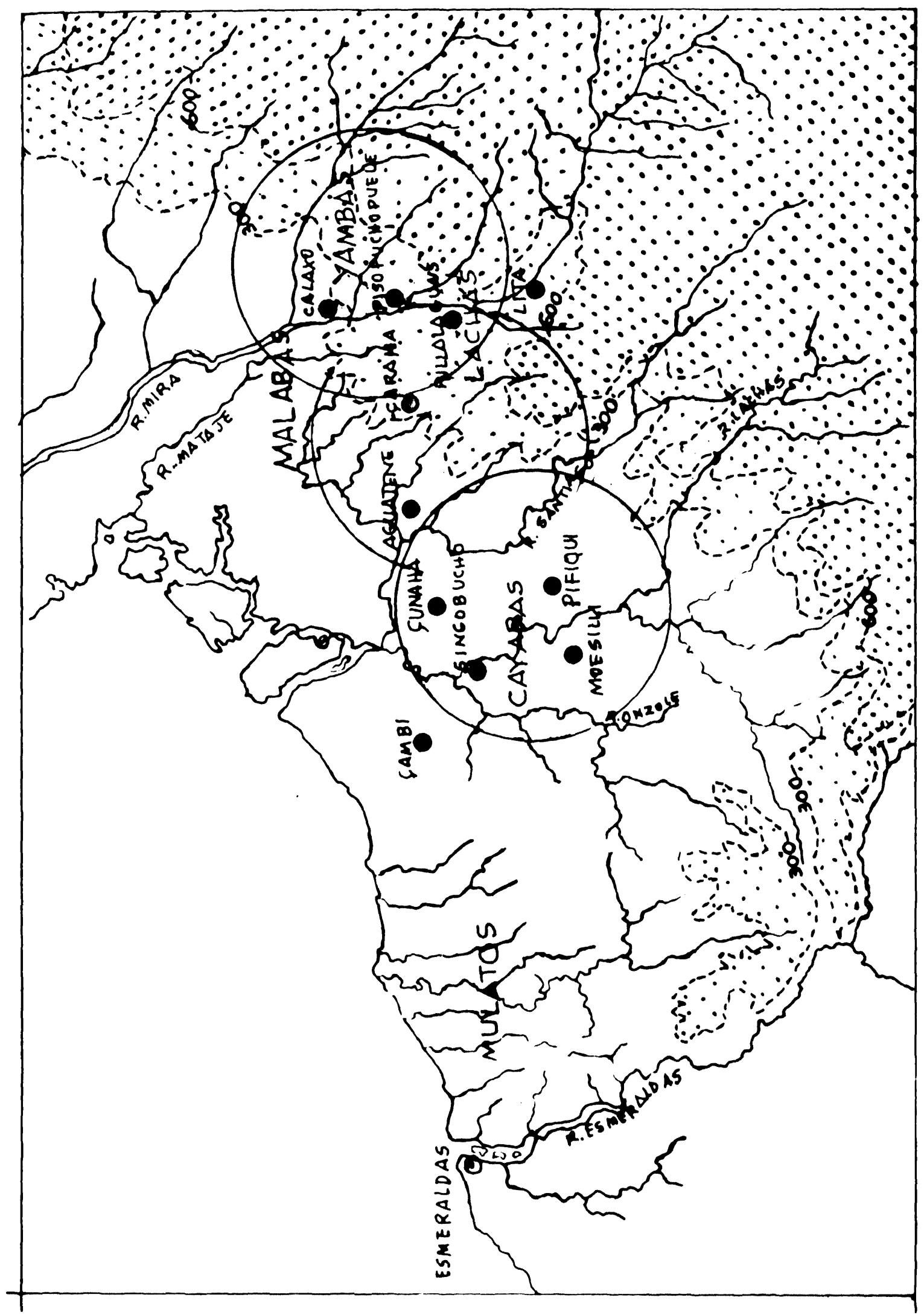


Nombres malabas: mujeres (continuación)

$\begin{array}{lcccccccrr}\text { Nombres } & 10 & 12 & 13 & 11 & 15 & 19 & 20 & 21 & \text { T'otal } \\ \text { Paçical } & 1 & & & & & & & \\ \text { Pascal } & & & & 1 & & & & \\ \text { Pascay } & & & & & 1 & & & 1 \\ \text { Pasque } & & & & & 1 & & & 1 \\ \text { Pasquiba } & & & & & 2 & & & 1 \\ \text { Tanfila } & & & & & & 1 & 1 \\ \text { Ugala } & 1 & & & & & & & 1 \\ \text { TO'TALES } & 11 & 1 & 3 & 22 & 14 & 1 & \\ \end{array}$

En la región intermedia comprendida entre el río Santiago y el río Mira, según ya dijimos anteriormente, hay una serie de ayllus en los que apreciamos un mcstizaje más o menos intenso. Los ayllus 6, 7 y 8 de Juan Aguazami, Juan Yahatino y Diego Zami se localizaban en la zona de Aguatene el ayllu 9 de Martín Nagola se hallaba en territorio de Caraha y el ayllu 11 de Diego Oñapapa no ha podido ser localizado.

En estos cinco ayllus apreciamos que las mujeres siempre llevan nombres cayapas, mientras los hombres, salvo en el ayllu 6 de Juan Aguazami, en el que dominan también los nombres cayapas, se reparten entre nombre de este grupo y otros totalmente diferentes en una proporción del 50 por ciento. Lo curioso es que estos últimos nombres que podrían corresponder a la etnia lacha, nunca se repiten ni dentro de un ayllu, ni en el conjunto de los cinco ayllus que estamos examinando.

A continuación damos la lista de estos nombres «extranjeros» con indicación numérica del ayllu en que aparecen: 
Aguahho (6)

Aguapeuro (6)

Aigo (9)

Cadllupo (9)

Cadmiso (8)

Calala (6)

Çalachuqueme (9)

Calunapumi (7)

Callembona (6)

Cança (9)

Capaquerami (8)

Carafa (6)

Caripicho (11)

Carium (6)

Cayabulo (6)

Chantere (6)

Chibusso (7)

Chillo (9)

Chiringuimi (7)

Chullamisso (7)

Chumu (7))

Cudinda (7)

Cugaono (11)

Cuyoapa (6)

Guapanaim (7)

Guaquena (7)

Hamelomi (8)

Hurenqueme (8)

Hullio (8)

Huro (8)

Huroquemi (9)

Huzala (11)

Lachapuento (9)

Lagara (6)

Laqueoro (9) 
Lusma (9)

Lluchucho (9)

Logoço (7)

Mallegauro (7)

Manamogo (9)

Manquiça (8)

Melpanbi (8)

Meguemi (9)

Minzala (8)

Miñufique (7)

Moguello (9)

Mogochidi (6)

Mogona (7)

Nafinchucho (6)

Nagola (9)

Nantaballo (7)

Napinche (8)

Napumi (8)

Nemid Peoron (6)

Nemuçe (6)

Nipayo (7)

Oyana (7)

Patahudpumi (6)

Pibeltuto (7)

Pidbuso (7)

Pidtulli (7)

Pidpeuro (7)

Pimagi (9)

Pimugo (6)

Pompona (6)

Puento (9)

Puezama (9)

Punpincho (7)

Purotuze (6)

Quicha (8)

Tomo XLVII 
Sapiuro (7)

Siuiculli (8)

Simbomogo (11)

Sumam (6)

Supegueme (9)

Supiama (6)

Susocan (11)

Susunga (9)

Tepecuya (6)

Tatago (9)

Tepuezuma (9)

Tualla (6)

Tuisullo (7)

Tullichurro (6)

Tunchi (6)

Tupalla (9)

Tutanqueme (7)

Ulchua (6)

Ulmio (6)

Urana (8)

Villegapa (9)

Yahafino (7)

Ymbapa (9)

Ynquegua (7)

Yza (8)

Zami (8)

Zibillo (7).

Los expresados son 97 nombres diferentes utilizados por hombres de los ayllus; 6: Juan Aguazami; 7: Juan Yahatino; 8: Diego Zami; 9: Martín Nagola y 11: Diego Oñapapa.

Algo parecido sucede en el caso de los ayllus 16, 17 y 18, de Diego Aguapapa, Sancho Aguatata y Martín Nagola, en los que una parte de la población corresponde, sin duda, al grupo cayapa, con nombres tan comunes como Aguapapa, Napapa, Aguamama, 
Naamama y Oñamama junto a los que aparecen otros que podrían corresponder a la etnia de los Yambas. En las tablas 9 y 10 se relicionan los nombres de hombres y mujeres.

TABLA 9

\begin{tabular}{|c|c|c|c|c|}
\hline Nombres & 16 & 17 & 18 & Total \\
\hline Caçiba & & & 1 & 1 \\
\hline Çima & & 1 & & 1 \\
\hline Cosemallapa & & & 1 & 1 \\
\hline Lillapata & & 1 & & 1 \\
\hline Llilliapapa & & 1 & & 1 \\
\hline Malaçapa & & & 1 & 1 \\
\hline Naagola & 1 & & 2 & 3 \\
\hline Nabgola & & & 1 & 1 \\
\hline Naçago & & & 2 & 2 \\
\hline Naçagua & & & 1 & 1 \\
\hline Naçona & & 1 & & 1 \\
\hline Nahapapa & 8 & 8 & & 16 \\
\hline Nalaçapa & & 2 & 1 & 3 \\
\hline Nalaque & & 1 & & 1 \\
\hline Napera & & 1 & & 1 \\
\hline Napilla & & 1 & 2 & 3 \\
\hline Omeçago & & & 1 & 1 \\
\hline ()ñacago & & & 1 & 1 \\
\hline Pilla & & & 2 & 2 \\
\hline Pillapapa & & & 1 & 1 \\
\hline Yçamogo & & 1 & & 1 \\
\hline Ysugami & & 1 & & 1 \\
\hline Zulli & & & 2 & 2 \\
\hline (OTAIES & 9 & 19 & 19 & 47 \\
\hline
\end{tabular}


TABLA 10

\begin{tabular}{lccc} 
Nombres & 17 & 18 & Total \\
Quiçona & 3 & 1 & 4 \\
Laquiçona & 2 & 3 & 5 \\
Maquiçona & & 1 & 1 \\
Naquiçona & & 2 & 2 \\
\multicolumn{1}{c}{ TOTALES } & 5 & 7 & 12
\end{tabular}

\section{CONCLuSión}

Del análisis al que hemos sometido el documento en el que se reproducen las listas de bautismos practicados por fray Gaspar de Torres en el territorio de los cayapas y sus vecinos podemos concluir que las dos etnias principales eran la de los cayapas y la de los malabas, situada la primera entre los ríos Onzole y Santiago y la segunda al sur de los ríos Mira y Mataje. El territorio entre ambos grupos estaba dominado en gran medida por los cayapas aunque en los ayllus correspondientes a este sector se aprecia un doble mestizaje con grupos que cabría identificar como lachas y yambas situados a ambos lados del curso alto del río Mira, camino de Lita.

Josefina Palop Martínez 